\title{
Experimental study of Ohmic losses of polarizer mirror system
}

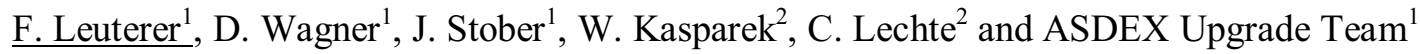 \\ ${ }^{1}$ Max Planck Institut für Plasmaphysik, 85748 Garching, Germany \\ ${ }^{2}$ Institut für Grenzflächenverfahrenstechnik und Plasmatechnologie, Universität Stuttgart, 70569 Stuttgart, Germany
}

Electron cyclotron heating of fusion plasmas requires to launch a high power millimeter wave beam with a well defined polarization, generally elliptical. This can be achieved with two corrugated mirrors with groove depths of $\approx \lambda / 8$ and $\approx \lambda / 4$. In HE11 waveguide transmission lines these polarizers are incorporated into $90^{\circ}$ miter bends and are subject to very high power density. To study their loss behaviour we have inserted into the ASDEX Upgrade ECRH transmission line such polarizers with sinusoidal grooves of periodicity $1.07 \mathrm{~mm}$, depth $0.8 \mathrm{~mm}$ $(\approx \lambda / 4$-mirror) resp. $0.57 \mathrm{~mm}(\approx \lambda / 8$-mirror $)$, and a thickness of $5 \mathrm{~mm}$. As material we took stainless steel with higher loss and easily measureable temperature rise, measured with a Pt-100 sensor on the backside of the mirror. The measurements were done with $50 \mathrm{~ms}$ pulses of $375 \mathrm{~kW}$ at $140 \mathrm{GHz}$. Between the output of the gyrotron and the mirror under test we have a quasi-optical MOU allowing to feed the waveguide with an arbitrary polarization.

We measured the losses of such polarizers and of a plane mirror depending on the angle $\varphi$ of a linearly polarized incident electric field, and on the angle $\alpha$ of the grooves, both with respect to the incidence plane of the miter bend. These are shown in Fig. 1. For a plane mirror and for $\varphi=0^{\circ}$ and $90^{\circ}$ we have theoretical results

$$
a_{\varphi=0^{\circ}}=a_{\perp} / \cos \Theta ; a_{\varphi=90^{\circ}}=a_{\perp} \cdot \cos \Theta ;
$$

where $\Theta=45^{\circ}$ is the angle of incidence in a miter bend mirror, and $a_{\perp}=4 R_{S} / Z_{0}$ is the absorption coefficient at perpendicular incidence with $R_{\mathrm{S}}$ being the surface resistance and $Z_{0}$ the free space wave impedance. From Fig. 1 top we get $a_{\perp}$ corresponding to $573 \mathrm{mV}$. This allows to normalize our measurements to $a_{\perp}$.

The measurements show a $\cos (2 \varphi)$, resp. $\cos (2 \alpha)$ dependence of the losses and suggest an empirical relation

$$
a_{\text {norm }}(\alpha, \varphi)=A E_{x}^{2}+B E_{z}^{2}+C H_{x}^{2}+D H_{z}^{2}
$$

with $E_{\mathrm{x}}, E_{\mathrm{z}}, H_{\mathrm{x}}, H_{\mathrm{z}}$ being the tangential electric and magnetic field components of the incident wave on the mirror surface perpendicular (x) and parallel (z) to the grooves. For a $90^{\circ}$ miter bend these are

$$
\begin{aligned}
& E_{x}=E_{0} \cos \alpha \sin \varphi+0.5 \sqrt{2} E_{0} \sin \alpha \cos \varphi \\
& E_{z}=E_{0} \sin \alpha \sin \varphi-0.5 \sqrt{2} E_{0} \cos \alpha \cos \varphi \\
& H_{x}=H_{0} \cos \alpha \cos \varphi-0.5 \sqrt{2} H_{0} \sin \alpha \sin \varphi \\
& H_{z}=H_{0} \sin \alpha \cos \varphi+0.5 \sqrt{2} H_{0} \cos \alpha \cos \varphi
\end{aligned}
$$

With measurements at $\alpha=0^{\circ}$ and $90^{\circ}$ and $\varphi=0^{\circ}$ and $90^{\circ}$ we can determine the coefficients $A, B, C$ and $D$. The lines in Fig. 1 and 3 are calculated with equ. (1) and fit very well to the measurements.

This empirical model allows to extend our AUGpolarization code to calculate for a given mirror arrange- ment and a given input polarization not only the output polarization, but also the loss. An example is shown in Fig. 2 displaying for a horizontal $\left(\varphi=0^{\circ}\right)$ linearly polarized input electric field the output ellipticity $\varepsilon$, the orientation $\beta$ of the ellipse, the sense of rotation $r$ of the polarization, and the total loss as function of the mirror rotation angles $\alpha_{\lambda / 8}$ and $\alpha_{\lambda / 4}$. The dots show a $\beta$-scan for a constant ellipticity $\varepsilon=0.2$ and rotation $r=+1$. Such calculations show that there are up to 4 possible settings $\alpha_{\lambda / 8}$ and $\alpha_{\lambda / 4}$ to realize a wanted output polatization as indicated in Fig. 2 by the black dots for an output polarization with $\beta=30^{\circ}$. These 4 settings are seen to have different loss.

To demonstrate this we selected some examples with different input and output polarizations and determined the losses for the 4 different settings. Some results are shown in Fig. 3.
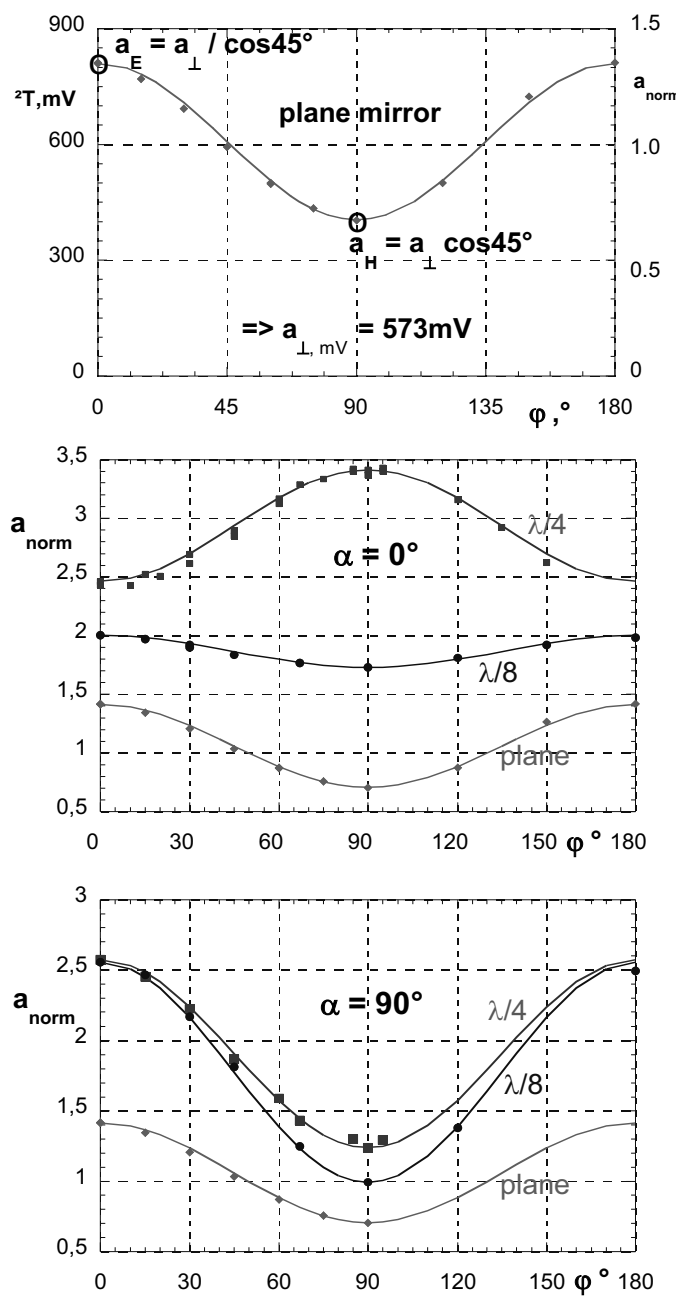

Fig. 1. measured and modelled losses of a plane, $\lambda / 8$ and $\lambda / 4$ mirrors as function of incidence angle $\varphi$ of linear polarization at constant groove orientation $\alpha$ 

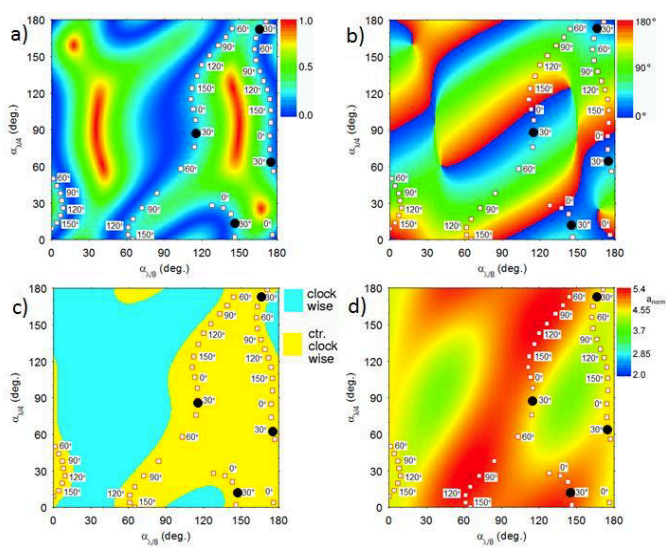

Fig. 2. polarization code result for linear horizontal input displaying a) ellipticity, b) orientation of the ellipse, c) rotation and d) loss as function of the groove angles $\alpha$
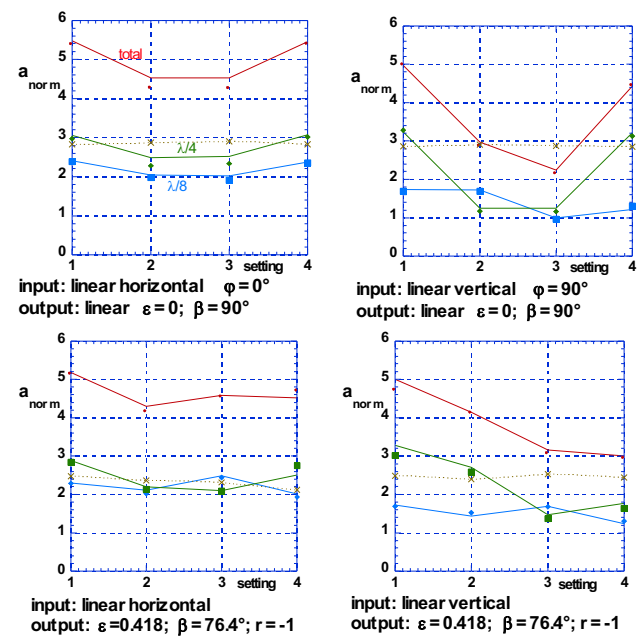

Fig. 3. Measured and calculated losses for the 4 possible settings leading to the same output polarization. Dots: total loss, squares: $\lambda / 4$-mirror, rhombs: $\lambda / 8$-mirror, crosses: detector signal at the end of the transmission line

We see that the loss can vary up to a factor of 2.5 depending on the setting of the individual mirrors. The constant detector signals indicate that indeed we have the same output polarization.
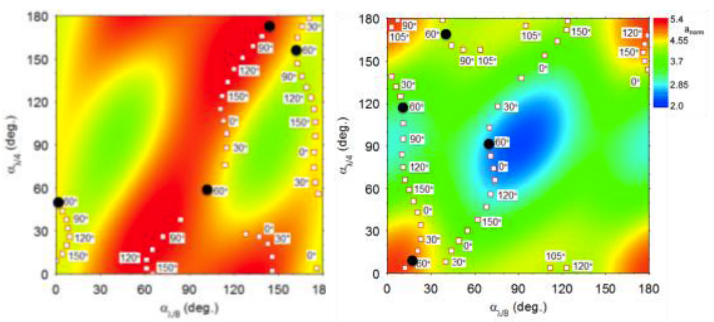

Fig. 4. polarizer loss as function of $\alpha_{\lambda / 8}$ and $\alpha_{\lambda / 4}$. Dots show a $\beta$-scan at const. ellipticity $\varepsilon=0.2$ and $r=+1$. Black dots show the 4 solutions for $\beta=60^{\circ}$. Input polarization horizontal $\varphi=0^{\circ}$ (left) and vertical $\varphi=90^{\circ}$ (right)

The total loss, i.e. the sum of the losses of both mirrors, depends on the geometrical arrangement of the mirrors and also on the input polarization. In Fig. 4 the two polarizers have the same incidence plane, but the linear input polarization is horizontal or vertical. In this example the vertical input polarization leads to much lower losses. An example for the dependence on the geometrical arrangement is shown in Fig. 5 where we assumed or- thogonal incidence of the two miter bends. Here too, the loss can be very different.
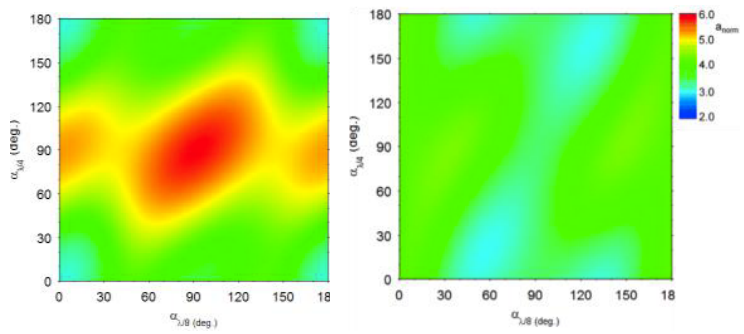

Fig. 5. polarizer loss as function of $\alpha_{\lambda / 8}$ and $\alpha_{\lambda / 4}$. In this case the incidence planes of the $\lambda / 4$ and the $\lambda / 8$ mirrors are perpendicular to each other. Input polarization: left horizontal $\varphi=0^{\circ}$, and right vertical $\varphi=90^{\circ}$

Arbitrary polarization can also be realized with a two mirror polarizer consisting of two $\lambda / 8$-mirrors instead of one $\lambda / 8$ and one $\lambda / 4$ mirror. We have repeated the set of measurements of Fig. 3 with such a configuration. The result is shown in Fig.6. In some cases even lower loss can be realized. Such a configuration has also the advantage that only one type of grooved mirror needs to be manufactured.
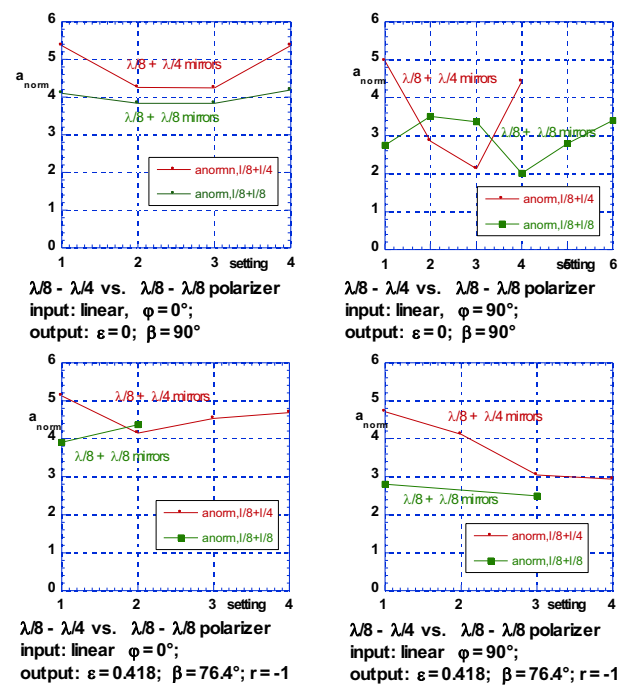

Fig. 6. measured losses of polarizer sets consisting of one $\lambda / 8$ and one $\lambda / 4$ mirror (dots) compared to a set with two $\lambda / 8$ mirrors

In this paper we have shown that the loss of a grooved mirror as used for polarizer systems is determined by just 4 parameters $A, B, C, D$, which can be obtained experimentally (or numerically) and which depend only on the corrugation profile. Knowing these parameters the losses of any arrangement of a polarizer system can be calculated. There are several possible settings for one wanted output polarization. These can have differences in the loss up to a factor of 2.5. Minimum loss may enhance the power transmission capability of the waveguide line.

1. D. Wagner, F. Leuterer, Broadband polarizers for high power multi-frequency ECRH systems, Journal of Infrared, Millimeter and Terahertz Waves, Vol. 26 (2005), 163-172.

2. D. Wagner, F. Leuterer et al., Minimization of the Ohmic loss of grooved polarizer mirrors in high power ECRH systems, Journal of Infrared, Millimeter and Terahertz Waves, Vol. 38 (2017), 191-205. 